\title{
Conservative Treatment with Continuous Cryotherapy as a Japanese Judo Therapy for Jones Fracture: A Case Report
}

\section{Takehito Sugasawa $^{1^{*} \dagger}$, Yasuko Yoshida ${ }^{2 \dagger}$, Katsuyuki Tokinoya ${ }^{2}$, Kentaro Ono $^{3}$ and Kazuhiro Takekoshi ${ }^{1}$}

${ }^{1}$ Division of Clinical Medicine, Faculty of Medicine, University of Tsukuba, 1-1-1 Tennodai, Tsukuba, Ibaraki 305-8577, Japan

${ }^{2}$ Department of Sports Medicine, Graduate School of Comprehensive Human Sciences, University of Tsukuba, 1-1-1 Tennodai, Tsukuba, Ibaraki 305-8577, Japan

${ }^{3}$ Department of Clinical Sciences, Graduate School of Comprehensive Human Sciences, University of Tsukuba, 1-1-1 Tennodai, Tsukuba, Ibaraki 305-8577, Japan

tThese authors contributed equally to this work as the first authors.

*Corresponding author: Takehito Sugasawa, Division of Clinical Medicine, Faculty of Medicine, University of Tsukuba, 235 Institutes of Medicine Building, 1-1-1 Tennodai, Tsukuba, Ibaraki 305-8577, Japan, Tel: +8190 44503521; E-mail: take0716@krf.biglobe.ne.jp

Rec date: June 19, 2017; Acc date: August 22, 2017; Pub date: August 24, 2017

Copyright: () 2017 Sugasawa T, et al. This is an open-access article distributed under the terms of the creative commons attribution license, which permits unrestricted use, distribution, and reproduction in any medium, provided the original author and source are credited.

\begin{abstract}
Introduction: Cryotherapy has been widely used as an acute treatment for traumatic injuries. However, it is rarely continuously used for fracture treatment for several weeks, particularly for refractory fractures. Some judo therapists, who can treat traumatic injuries under the Japanese law, use cryotherapy as a continuous treatment for fractures. Herein, we report a case of Jones fracture (refractory) treated by a therapist using continuous cryotherapy.
\end{abstract}

Case presentation: A 49-year-old woman had a non-transposable Jones fracture located at the metaphysis of the right 5th metatarsal from an inversion injury in the ankle. At this time, the patient had severe pain and swelling, and her walking ability was impaired. Three days after the fracture, the therapist started conservative treatment mainly using cryotherapy for the lesion part for 15 min thrice every day; splint-fixation using a hard cardboard was then performed using the standard style of the Japanese Judo therapy.

Fifteen days after the fracture, the numerical rating scale (NRS) score of the patient notably decreased to 1.5 compared with the first NRS score of 10 . Moreover, pain upon normal walking with full-weight bearing completely resolved, and the ankle had a full range of motion. Moreover, there was no muscle atrophy.

Twenty-four days after the fracture following daily cryotherapy, the NRS score was 0 , and there were no problems in the performance of activities of daily living and work. Moreover, mild running and going up and down the stairs became possible.

Finally, the patient showed excellent clinical and functional results at the two-month and one-week follow-up, and the side effects of this therapy were not observed.

Conclusion: This case highlights the use of cryotherapy as a continuous treatment for Jones fracture.

Keywords: Cryotherapy; Jones fracture; Refractory fracture; Japanese judo therapy

\section{Introduction}

Cryotherapy is a widely used therapeutic strategy for injuries such as fractures, dislocations, sprains and pulled muscles [1]. However, previous studies revealed contradicting results regarding its efficacy for tissue injuries in vivo [2-5].

Bleakley [6] conducted the only systematic review on cryotherapy for soft tissue injuries. They concluded that the exact efficacy of cryotherapy remains unclear and that additional high-quality studies are required to elucidate this issue [6].

Although the efficacy of cryotherapy has remained unclear, therapists who believe that cryotherapy can prevent exacerbations of injuries and inflammations have still used it as an acute treatment for various injuries, particularly in the soft tissues. Additionally, it is clinically rare that it is continuously used as a fracture treatment for several weeks after the acute phase.

Judo therapists, who can treat traumatic injuries without surgery under the Japanese law, have sometimes treated fractures using cryotherapy as a continuous treatment for several weeks. Although there are many contradicting findings in the use of cryotherapy as a continuous treatment for various injuries in the clinical setting, some Judo therapists still use it. Moreover, Judo therapists often observe that the patients' satisfaction is high when it is continuously performed for several weeks.

Jones fracture is defined as an acute fracture and is located at the metaphysis $1.5 \mathrm{~cm}$ to $2 \mathrm{~cm}$ from the proximal end of the 5th metatarsal, which commonly occurs from an inversion injury in the ankle [7]. Jones fracture is also classified as a zone 2 fracture in the classification of Lawrence and Botte [8]. Zone 2 fractures are known to have a higher chance of non-healing (nonunion) and risk of re-fracture 
Citation: Sugasawa T, Yoshida Y, Tokinoya K, Ono K, Takekoshi K (2017) Conservative Treatment with Continuous Cryotherapy as a Japanese Judo Therapy for Jones Fracture: A Case Report. J Trauma Treat 6: 390. doi:10.4172/2167-1222.1000390

Page 2 of 5

even after healing [9]. Clinically, many patients with wide ranges of age in Japan experience this type of fracture, regardless of their sex.

Herein, we report a case of Jones fracture treated by a Japanese Judo therapist using continuous cryotherapy for several weeks; this could be the first report to use continuous cryotherapy for Jones fracture as a refractory injury.

\section{Case Presentation}

\section{Patient's history}

A 49-year-old woman presented with the following history. She had ankle sprain of inversion, going down the stairs. After one day, she had a medical examination (radiography, etc.) by a Japanese orthopedist and was diagnosed with a fracture at the metaphysis of the right 5th metatarsal, i.e., Jones fracture from an acute inversion injury, classified as a zone 2 fracture in the classification of Lawrence and Botte [8] (Figure 1). She also had present illnesses, which included menopausal syndrome, renal disturbance-associated proteinuria, and class 1 obesity. However, she did not take specific medications for these illnesses.

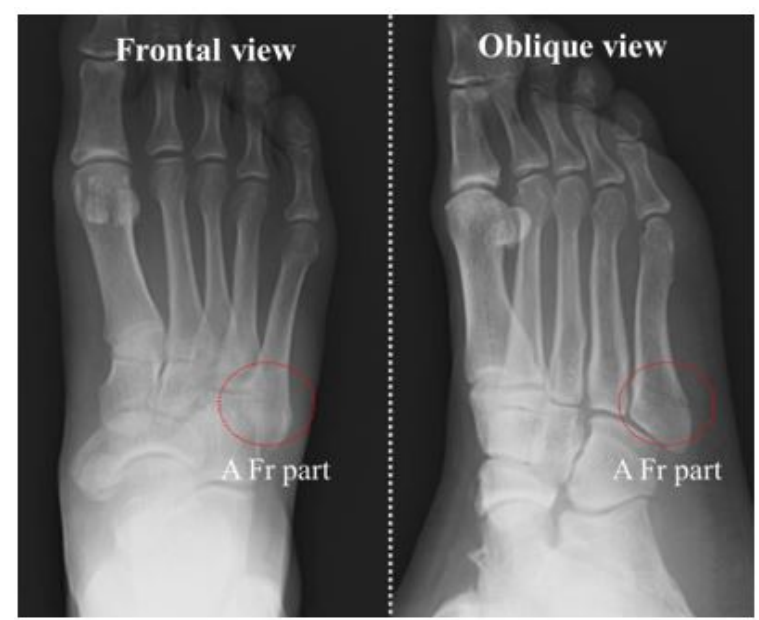

Figure 1: Radiographic image of the patient's right foot one day after the fracture. The image shows a Jones fracture, classified as a zone 2 fracture on the classification of Lawrence and Botte [8].

\section{Examinations by a Judo therapist}

Three days after the fracture, a Judo therapist clinically observed her condition again. She complained of more severe pain (numerical rating scale (NRS) score [10], swelling, and subcutaneous bleeding on the foot than those experienced at the first day of the fracture. Objectively, these symptoms were observed on the fractured foot as shown in Table 1. Table 1 shows the summarized temporal symptoms and treatments. Therefore, the therapist started treatments using cryotherapy and external fixation with the agreement of a medical doctor.

\section{Cryotherapy by the Judo therapist}

Cryotherapy was performed for the lesion part with mild compression using a bandage and an ice bag with a water:ice ratio of $1: 1$; the surface temperature was $3.2^{\circ} \mathrm{C}$. Cryotherapy was performed for
15 min thrice every day (Figure 2). Simultaneously, splint-fixation using a hard cardboard combined with a non-elastic bandage on the foot to the ankle was performed, which is the standard style in the Judo therapy (Figure 3). This fixation enabled walking and external rotation of the limb. Additionally, it was mended in accordance with the reduction of the swelling on each day.

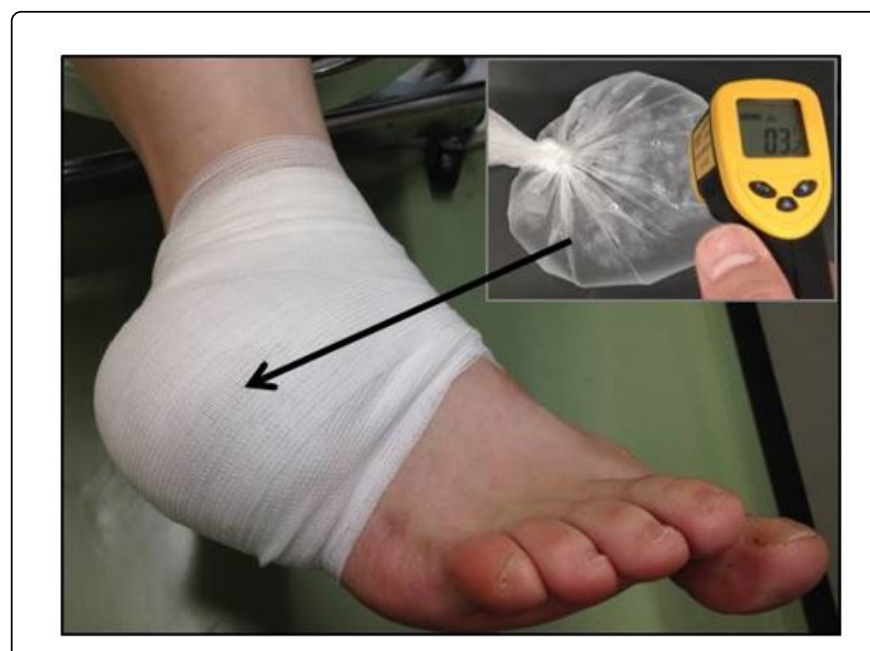

Figure 2: Method of the cryotherapy. Cryotherapy is performed using an ice bag wrapped by elastic bandage.

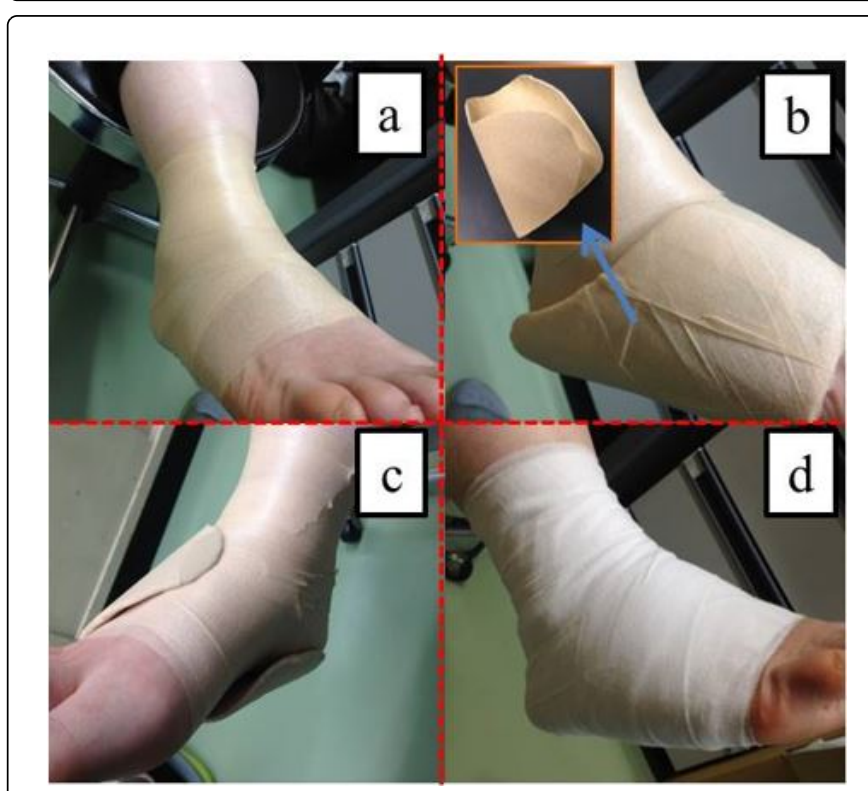

Figure 3: Fixation using a hard cardboard and non-elastic bandage. The fixation is performed as follows: a) Underlap taping to protect the skin; b) and c) hard cardboard covered and non-covered by the underlap tape; and d) the fixation is finished using a non-elastic bandage.

The therapist also discussed to the patient the following six important measures: 1) One-sided crutch should be used if frequent walking is required at work; 2) removing the fixation is only allowed when the patient has to take a bath and should be done carefully; however, this fixation must be worn again after taking a bath; 3) the 
Citation: Sugasawa T, Yoshida Y, Tokinoya K, Ono K, Takekoshi K (2017) Conservative Treatment with Continuous Cryotherapy as a Japanese Judo Therapy for Jones Fracture: A Case Report. J Trauma Treat 6: 390. doi:10.4172/2167-1222.1000390

Page 3 of 5

foot and fingers should be well washed to protect them against tinea during the bath; 4) vitamin C $1000 \mathrm{mg}$ should be taken daily to prevent complex regional pain syndrome type $1(\mathrm{CRPS} 1)$; 5) activities that stimulate pain should be avoided for a normal healing process; however, activities of daily living should be maintained as long as no pain occurs; and 6) cryotherapy should be performed by the patient herself every day even in times when the therapist has not performed it. The patient agreed to perform all the above-mentioned measures.

\section{Follow-up at six days after the fracture}

This follow-up was conducted three days after the cryotherapy started. The NRS score markedly decreased to 3 and was objectively observed on the fractured foot as shown in Table 1.

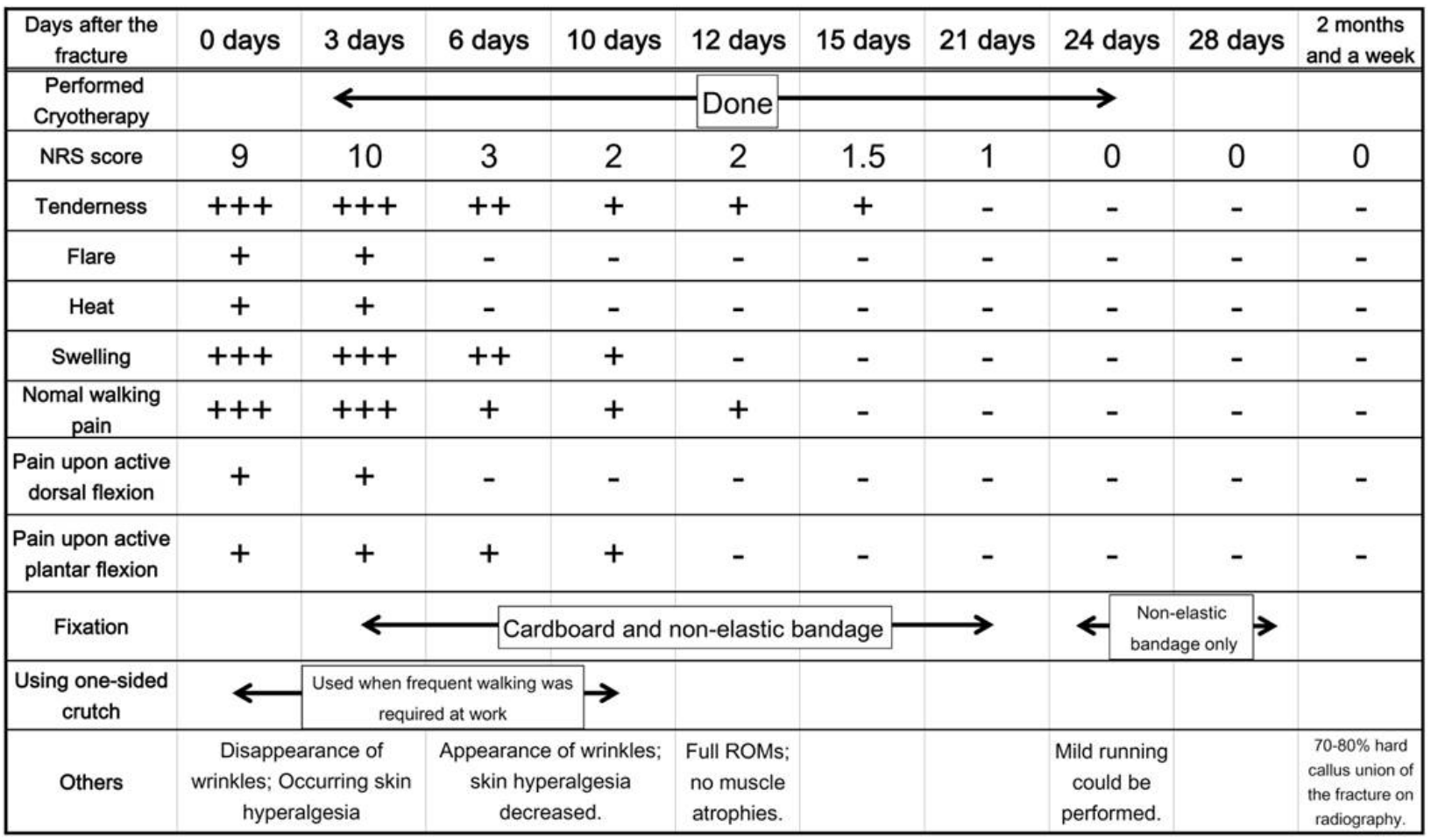

NRS, numerical rating scale; ROM, range of motion

Table 1: Summarized temporal symptoms and treatments of the patient.

Additionally, she could walk without using a one-sided crutch with mild pain. However, the pain worsened after finishing work. Therefore, the therapist instructed the patient that if pain occurs, cryotherapy should be performed, and if it does not occur during walking, removing the one-sided crutch is possible. She also agreed to perform all the above-mentioned measures. Moreover, dorsal flexion exercise was also started to prevent re-injury.

\section{Follow-up at ten days after the fracture}

This follow-up was conducted seven days after the cryotherapy started. The NRS score further decreased to 2 and was objectively observed on the fractured foot as shown in Table 1.

Additionally, she could already walk without using the one-sided crutch and step on the stairs, since pain upon walking and external limb rotation was non-existent or was only mild. Therefore, the therapist allowed the removal of the one-sided crutch since this day.

\section{Follow-up at 12 days after the fracture}

This follow-up was conducted nine days after the cryotherapy started. The NRS score was 2 , which was similar to that at 10 days after the fracture; it was objectively observed on the fractured foot as shown in Table 1.

Moreover, the therapist checked the ranges of motion (ROMs) of both the ankle and foot fingers; they had full ROMs, and arthrogryposis and muscle atrophies were not observed. Additionally, she experienced no pain upon external rotation while walking; however, she experienced mild pain and insecurity when stepping on the stairs. Consequently, she verbalized that her mental condition was very good because she was almost able to return to her original activities of daily living and working. Therefore, the therapist allowed normal walking with full-weight bearing in the non-painful range and removal of the fixation temporarily while resting in her home or working place (Figure 4a). 


\section{Follow-up at 15 days after the fracture}

This follow-up was conducted 12 days after the cryotherapy started. The NRS score further decreased to 1.5 and was objectively observed on the fractured foot as shown in Table 1. The radiographic image is shown in Figure $4 \mathrm{~b}$.

Additionally, the appearance of the injured foot was almost normal, and her limb externally rotated owing to her sense of uneasiness only upon stepping on the stairs; however, pain upon normal walking with full-weight bearing completely resolved. Surprisingly, it seemed that the fracture spread out on the radiographic image as shown in Figure $4 \mathrm{~b}$, even though the symptoms, especially pain, almost resolved.

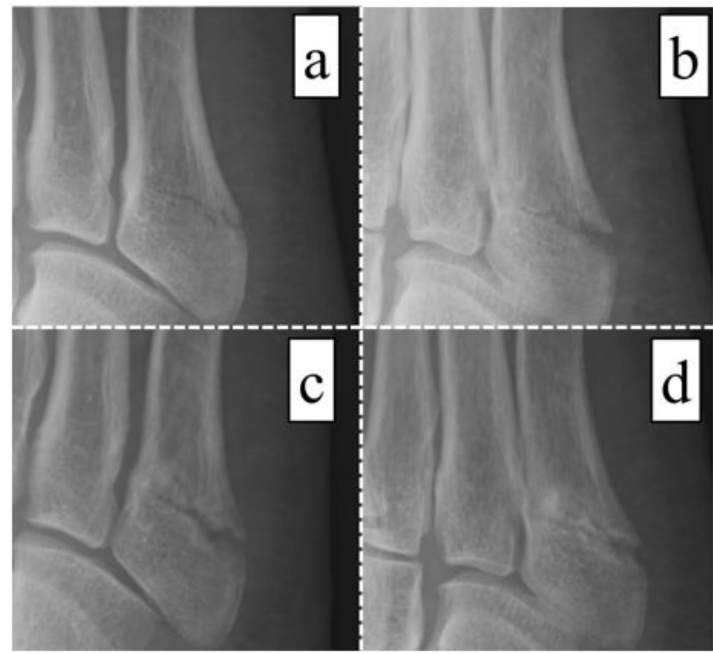

Figure 4: Temporal radiographic image of the fracture site a) 1 day, b) 15 days, c) 28 days, and d) 2 months and a week after the fracture.

\section{Follow-up at 21 days after the fracture}

This follow-up was conducted 18 days after the cryotherapy started. The NRS score further decreased to 1 , and it was objectively observed that the tenderness in the fracture site completely resolved (Table 1). Additionally, she could stand on tiptoes on both feet and could already remove the fixation by herself, since the pain was almost resolved, and she did not feel any insecurity even while walking normally and stepping on the stairs; however, the therapist mentioned the possibility of re-injury to the patient. Therefore, the fixation was modified to a non-elastic bandage without a cardboard, enveloping the foot and the ankle, which was only used at her working place.

\section{Follow-up at 24 days after the fracture}

This follow-up was conducted 21 days after the cryotherapy started. The NRS score further decreased to 0 ; she could already perform mild running (Table 1) and did not feel any pain and insecurity during activities of daily living and working. Therefore, the therapist modified the method of cryotherapy in which it was only performed when the patient felt pain. Additionally, the patient performed and followed the important measures discussed by the therapist well until this time.

\section{Follow-up at 28 days after the fracture}

At this time, the patient had a better condition, and the non-elastic bandage was completely removed. Moreover, the radiographic images showed a hard callus formation around the fracture site (Figure 4c).

\section{Follow-up at 2 months and a week after the fracture}

The patient did not have any complaints and verbalized that the therapies had better effects than the standard treatment. Moreover, the radiographic images showed a $70-80 \%$ union by a hard callus at the fracture site (Figure $4 \mathrm{~d}$ ).

\section{Discussion}

Although cryotherapy has been widely used for acute traumatic soft tissue injuries, this case used cryotherapy for Jones fracture. Therefore, this report could be the first to use continuous cryotherapy as a rare treatment for Jones fracture as a refractory injury. In this case, the patient felt that cryotherapy was effective treatment because there were no muscle atrophy and joint contracture even when the fixations were removed; further, her NRS score decreased immediately, allowing normal walking and return to original activities of daily living and working without pain at 12 days after the fracture. Moreover, the radiographic images at 2 months and a week showed a $70 \%$ to $80 \%$ union by a hard callus. These results are better than previously reported results on Jones fracture using conservative treatments $[10,11]$. However, highly detailed mechanisms of these phenomena are unknown.

Considering the results of a previous study, a hypothesis can be made. For skin wounds, adenosine triphosphate (ATP) addition promotes the healing process in vivo [12]; we previously reported that optimal cold stimulations increase mitochondrial activity and cellular ATP concentration in an in vitro study [1]. Moreover, mitochondrial dysfunction impeding ATP production occurs in the impaired tissues in CRPS1 [13], which often occurs after fracture and prolongs the healing process, associating long-term severe pain and non-union of fractured bones. Therefore, there is a possibility that cryotherapy contributes to the normal or accelerated fracture healing process via activations of the mitochondria and other factors. To verify this hypothesis, in vivo and in vitro studies may be required in detail.

At 15 days after the fracture, it seemed that the fracture site spread out on the radiographic image as shown in Figure $4 \mathrm{~b}$, even though the symptoms of the patient, especially pain, almost resolved. These phenomena are often observed in the healing process of adult fractures irrespective of the use of cryotherapy. It has been reported that osteoclasts in the healing process of fractures are absorbed in the damaged bones to rebuild new bones [14]. Therefore, it is possible that the spreading out of the fracture site on the radiographic image in this case results in osteoclast production to remove the damaged bone, which does not correlate to the patient's symptoms, especially pain.

\section{Conclusion}

The treatments in this case were successful, and the results were excellent. However, this case is only a single, which have a lot of limitations. We have tried further research to clarify the effect of cryotherapy on fracture. This report highlights the use of cryotherapy as a continuous treatment for Jones fracture. 
Citation: Sugasawa T, Yoshida Y, Tokinoya K, Ono K, Takekoshi K (2017) Conservative Treatment with Continuous Cryotherapy as a Japanese Judo Therapy for Jones Fracture: A Case Report. J Trauma Treat 6: 390. doi:10.4172/2167-1222.1000390

Page 5 of 5

\section{Ethical Consent}

We sent the article to the patient; she has read the article and has agreed with its publication. The patient declared that she does not have any problem in the publication of this article.

\section{Competing Interests}

The authors declare that there are no competing interests in this report.

\section{References}

1. Sugasawa T, Mukai N, Tamura K, Tamba T, Mori S, et al. (2016) Effects of cold stimulation on mitochondrial activity and VEGF expression in vitro. Int J Sports Med 37: 766-778.

2. Puntel GO, Carvalho NR, Amaral GP, Lobato LD, Silveira SO, et al. (2011) Therapeutic cold: An effective kind to modulate the oxidative damage resulting of a skeletal muscle contusion. Free Radic Res 45: 125-138.

3. Puntel GO, Carvalho NR, Dobrachinski F, Salgueiro AC, Puntel RL, et al. (2013) Cryotherapy reduces skeletal muscle damage after ischemia/ reperfusion in rats. J Anat 222: 223-230.

4. Takagi R, Fujita N, Arakawa T, Kawada S, Ishii N, et al. (2011) Influence of icing on muscle regeneration after crush injury to skeletal muscles in rats. J Appl Physiol 110: 382-388.

5. Nusair YM (2007) Local application of ice bags did not affect postoperative facial swelling after oral surgery in rabbits. Br J Oral Maxillofac 45: 48-50.
6. Bleakley C, McDonough S, MacAuley D (2004) The use of ice in the treatment of acute soft-tissue injury: A systematic review of randomized controlled trials. Am J Sports Med 32: 251-261.

7. Jones R (1902) Fracture of the base of the fifth metatarsal bone by indirect violence. Ann Surg 35: 697-700.

8. Lawrence SJ, Botte MJ (1993) Jones' fractures and related fractures of the proximal fifth metatarsal. Foot Ankle 14: 358-365.

9. The American Orthopaedic Foot and Ankle Society (2017) Treatments fifth metatarsal fracture surgery.

10. Chuckpaiwong B, Queen RM, Easley ME, Nunley JA (2008) Distinguishing Jones and proximal diaphyseal fractures of the fifth metatarsal. Clin Orthop Relat Res 466: 1966-1970.

11. Adhikari BR, Thakur R, Gurung G (2010) Comparative study of early screw fixation versus cast application on the treatment of acute Jones fracture 10: 4-9.

12. Wang J, Zhang Q, Wan R, Mo Y, Li M, et al. (2009) Intracellular adenosine triphosphate delivery enhanced skin wound healing in rabbits. Ann Plast Surg 62: 180-186.

13. Tan EC, Janssen AJ, Roestenberg P, Van Den Heuvel LP, Goris RJ, et al. (2011) Mitochondrial dysfunction in muscle tissue of complex regional pain syndrome type I patients. Eur J Pain 15: 708-715.

14. Vuolteenaho K, Moilanen T, Moilanen E (2008) Non-steroidal antiinflammatory drugs, cyclooxygenase- 2 and the bone healing process. Basic Clin Pharmacol Toxicol 102: 10-14. 